\title{
ON LEVI'S DUALITY BETWEEN PERMUTATIONS AND CONVERGENT SERIES
}

\author{
QUENTIN F. STOUT
}

\section{Introduction}

This paper concerns a duality between conditionally convergent real series and permutations of their indices. It is widely known that if one is given a series $\sum a_{i}$ which is conditionally convergent but not absolutely convergent, then there is a permutation $\pi$ such that $\sum a_{i} \neq \sum a_{\pi(i)}$. Various authors have studied extensions of this result of Riemann (for example, Smith [8], Steinitz [9], Threlfall [10], Wald [11, 12]), but in our opinion a more challenging problem is to find those permutations which do not change the value of $\Sigma a_{i}$, and, dually, given a permutation to find those series whose sum is unaffected by the permutation.

F. W. Levi [5] was apparently the first to consider such problems, and he introduced an interesting duality between subsets of $\mathbf{C}$, denoting the set of all convergent real series, and subsets of $\mathbf{P}$, denoting the set of permutations of the counting numbers $\mathbb{N}=\{1,2, \ldots\}$. Given a set $A \subseteq \mathbf{C}$, let

$$
A^{\times}=\left\{\pi \in \mathbf{P}: \Sigma a_{i}=\Sigma a_{\pi(i)} \text { for all } a \in A\right\},
$$

and given a $P \subseteq \mathbf{P}$, let

$$
P^{+}=\left\{\Sigma a_{i} \in \mathbf{C}: \Sigma a_{i}=\Sigma a_{\pi(i)} \text { for all } \pi \in P\right\} .
$$

Levi called $A^{\times+}$the closure of $A$, and $P^{+\times}$the closure of $P$, and noted that ${ }^{\times}$and + are inverses of each other when considered as maps between closed sets of series and closed sets of permutations. In particular, this duality can be used to show that the closed sets of permutations and series each form a lattice, where

$$
\begin{array}{ll}
A \vee B=\left(A^{\times} \cap B^{\times}\right)^{+}, & A \wedge B=\left(A^{\times} \cup B^{\times}\right)^{+}, \\
P \vee Q=\left(P^{+} \cap Q^{+}\right)^{\times}, & P \wedge Q=\left(P^{+} \cup Q^{+}\right)^{\times}
\end{array}
$$

for closed sets $A, B \subseteq \mathrm{C}$ and $P, Q \subseteq \mathbf{P}$. The ${ }^{\times}$and + maps are lattice antiisomorphisms, taking $\vee$ to $\wedge$ and vice versa.

One highly unusual property of this duality is that $\mathbf{C}$ is an unnormed linear space, while $\mathbf{P}$ has a natural multiplicative group structure. It is easily seen that every closed set of series is a linear subspace of $\mathbf{C}$, but it is rarely true that a closed set of permutations is a subgroup or even a subsemigroup. In fact, Levi showed that $\mathbf{P}$ is the only closed subgroup. He conjectured that for any convergent series $\Sigma a_{i}$ which is not absolutely convergent, $\left(\Sigma a_{i}\right)^{\times}$is not a semigroup, and asked if $\mathbf{P}$ and $\mathbf{C}^{\times}$were the only closed semigroups. Pleasants [6] and Smith [8] also consider several problems relating convergent series and the group structure of $\mathbf{P}$.

Received 22 August 1984; revised 12 March 1986.

1980 Mathematics Subject Classification 40A05. 
This paper examines some of the interplay between Levi's duality, the linearity of $\mathbf{C}$, and the group structure of $\mathbf{P}$. We answer Levi's question, and initiate a study of the structure of the lattices of closed subsets of $\mathbf{P}$ and $\mathbf{C}$. The paper is divided into six sections. The next section contains basic definitions and previous results. The third section examines the semigroup properties of large closed sets of permutations of the form $\left(\sum a_{i}\right)^{\times}$, and uses these results to answer Levi's question. The fourth section contains results on small closed sets of permutations, those of the form $\pi^{+x}$, concentrating on the structure of the sublattice of closed sets beneath $\pi^{+\times}$. The fifth section considers the set of alternating series, which is the most important class of conditionally convergent series, and characterizes its dual and second dual. The final section contains some open problems.

\section{Preliminaries}

For sets $A$ and $B, A \subset B$ (or $B \supset A$ ) will mean strict inclusion, that is, $A \subseteq B$ and $A \neq B$. The term conditionally convergent will mean convergent but not absolutely convergent, and $\mathbf{A C}$ will denote the set of absolutely convergent series. If the series have terms from some normed linear space, then $\mathbf{P}^{+}$is the set of unconditionally convergent series. One always has $\mathbf{P}^{+} \supseteq \mathbf{A C}$, and for finite-dimensional spaces (in particular, for the reals $\mathbb{R}), \mathbf{P}^{+}=\mathbf{A C}$. An important result of Dvoretzky and Rogers [2] is that in every infinite-dimensional Banach space $\mathbf{P}^{+} \neq \mathbf{A C}$. In this paper only real series will be considered.

We use $\mathbf{P}_{\mathbf{0}}$ to denote $\mathbf{C}^{\times}$. Despite the long-term interest of mathematicians in relating conditional convergence, absolute convergence and unconditional convergence, apparently the first characterization of $\mathbf{P}_{\mathbf{0}}$ is due to Levi [5]. Subsequently, Agnew [1] and Pleasants [6] independently characterized $\mathbf{P}_{\mathbf{0}}$. Goha [3] published an alternative proof of Levi's result, and Schaefer's expository paper [7] includes another characterization of $\mathbf{P}_{0}$. For $A \subseteq \mathbb{N}$, the phase ' $A$ is a union of $n$ intervals of $\mathbb{N}$ ', means that $A$ can be written as a union of $n$ intervals of $\mathbb{N}$, but cannot be written as a union of $n-1$ intervals. Here a single element of $\mathbb{N}$ is considered to be an interval. For integers $i \leqslant j,[i, j]$ denotes the interval $\{k \in \mathbb{N}: i \leqslant k \leqslant j\}$. For a permutation $\pi$ and an interval $[i, j], \pi([i, j])$ denotes the set $\{\pi(i), \ldots, \pi(j)\}$.

THeOREM 1 (Levi [5]). $\pi \in \mathbf{P}_{\mathbf{0}}$ if and only if there is an integer I such that, for all $n, \pi([1, n])$ is a union of $I$ or fewer intervals of $\mathbb{N}$.

From the definition of $\mathbf{P}_{\mathbf{0}}$ one knows only that for any permutation $\pi$ in $\mathbf{P} \backslash \mathbf{P}_{\mathbf{0}}$ there is a series $\Sigma a_{i}$ in $\mathbf{C}$ such that $\Sigma^{n} a_{\pi(i)}$ does not converge to the sum $\Sigma a_{i}$. (For summations, whenever a lower bound is omitted it is 1 , and whenever an upper bound is omitted it is infinity.) Levi showed that additionally one can find a convergent series $\Sigma b_{i}$ such that $\Sigma b_{\pi(i)}$ diverges.

Levi's closure operations have several simple properties. The set $\mathbf{C}$ is the largest closed set of series and $\mathbf{A C}$ is the smallest, just as $\mathbf{P}$ is the largest closed set of permutations and $\mathbf{P}_{0}$ is the smallest. For any $A \subseteq \mathbf{C}$, we have $A^{\times+} \supseteq \operatorname{span}(A \cup \mathbf{A C})$, where span $(X)$ is the linear span of $X$. Later sections contain examples where the closure is precisely this linear closure, and there are $A$ where $A^{\times+}$is strictly greater than span ( $A \cup \mathbf{A C}$ ). For example, if $A$ is the set of all square-summable convergent series then $\operatorname{span}(A \cup \mathbf{A C})=A \neq \mathbf{C}$, but $A^{\times+}=\mathrm{C}$. (This later fact may not be obvious, but Levi's proof of Theorem 1 can be modified to construct, for each 
$\pi \in \mathbf{P} \backslash \mathbf{P}_{0}$, a square-summable convergent series $\Sigma a_{i}$ such that $\Sigma a_{i} \notin \pi^{+}$.) Further, if $B$ and $C$ are closed sets of series, then $B \vee C \supseteq \operatorname{span}(B \cup C)$ and $B \wedge C=B \cap C$, and if $P$ and $Q$ are closed sets of permutations, then $P \vee Q \supseteq P \cup Q$ and $P \wedge Q=P \cap Q$.

Unfortunately, notation concerning permutations is not standardized. For example, the action of the permutations in Pleasant's paper [6] is the inverse of their action here. Given permutations $\pi$ and $\rho, \pi \circ \rho$ will mean the permutation given by $\pi \circ \rho(i)=\pi(\rho(i))$. For any $\pi$ in $\mathbf{P}$ and $\rho$ in $\mathbf{P}_{0}, \pi \circ \rho$ is in $\pi^{+\times}$, but in general $\rho \circ \pi$ is not in $\pi^{+\times}$. Some of the results concerning $\mathbf{C}$ and products of permutations follow. Note that $\mathbf{P}$ is a group and $\mathbf{P}_{0}$ is a semigroup.

TheOREM 2 (Levi [5]). P is the only closed set of permutations which is a group.

THEOREM 3 (Pleasants [6]). Every permutation is the product of two sum-preserving permutations, where a permutation $\pi$ is sum-preserving if, for each $\Sigma a_{i}$ in $\mathbf{C}$, either $\Sigma a_{i}=\Sigma a_{\pi(i)}$ or $\Sigma a_{\pi(i)}$ diverges.

TheOREM 4 (Pleasants [6]). The group generated by $\mathbf{P}_{0}$ is not all of $\mathbf{P}$.

Note that if $\pi$ moves only finitely many elements then, for any permutation $\rho$, $\rho^{-1} \circ \pi \circ \rho$ also moves only finitely many elements. In this case both $\pi$ and $\rho^{-1} \circ \pi \circ \rho$ are in $\mathbf{P}_{0}$, and hence in $\left(\Sigma a_{i}\right) \times$ for any series $\Sigma a_{i}$.

THEOREM 5 (Smith [8]). Let $\pi$ be a permutation which moves infinitely many elements, and let $\Sigma a_{i}$ be conditionally convergent. Then there are permutations $\rho_{1}$ and $\rho_{2}$ such that $\rho_{1}^{-1} \circ \pi \circ \rho_{1} \in\left(\Sigma a_{i}\right)^{\times}$and $\rho_{2}^{-1} \circ \pi \circ \rho_{2} \notin\left(\Sigma a_{i}\right)^{\times}$.

\section{3. $\left(\Sigma a_{i}\right)^{\times}$}

In this section we consider large closed sets of permutations, those of the form $\left(\Sigma a_{i}\right)^{\times}$. Basically, Theorem 7 shows that all such sets have certain common properties, while Theorem 9 shows that $\left(\Sigma a_{i}\right)^{\times}$provides detailed information about $\Sigma a_{i}$.

We say that $\Sigma a_{i}$ converges absolutely on $J \subseteq \mathbb{N}$ if $\sum_{j \in J}\left|a_{j}\right|<\infty$, and $\Sigma a_{i}$ is indeterminate on $J$ if $\Sigma_{j \in J} \max \left(0, a_{j}\right)=+\infty$ and $\Sigma_{j \in J} \min \left(0, a_{j}\right)=-\infty$. If $\Sigma a_{i}$ is conditionally convergent and converges absolutely on $J$ then it is indeterminate on $\mathbb{N} \backslash J$. Any convergent series has uncountably many infinite subsets of $\mathbb{N}$ on which it converges absolutely.

LEMMA 6. Let $\Sigma a_{i}$ be conditionally convergent, let J be a set on which $\Sigma a_{i}$ converges absolutely, and let $\pi \in \mathbf{P}$ be such that $\pi^{-1}(\mathbb{N} \backslash J)$ is monotone. Then $\pi \in\left(\Sigma a_{i}\right)^{\times}$.

Proof. Let $M(n)=\max \left\{i: i \in \mathbb{N} \backslash J\right.$ and $\left.\pi^{-1}(i) \leqslant n\right\}$. Then

$$
\begin{aligned}
\sum^{n} a_{\pi(i)}=\sum^{M(n)} a_{i} & +\Sigma\left\{a_{i}: i>M(n), i \in J, \pi^{-1}(i) \leqslant n\right\} \\
& -\Sigma\left\{a_{i}: i \leqslant M(n), i \in J, \pi^{-1}(i)>n\right\} .
\end{aligned}
$$

As $n \rightarrow \infty, M(n) \rightarrow \infty$, so the first sum on the right-hand side converges to $\Sigma a_{i}$. Since $\Sigma a_{i}$ converges absolutely on $J$, the remaining two sums on the right converge to 0 . Therefore the left-hand side converges to $\Sigma a_{i}$. 
THEOREM 7. Let $\Sigma a_{i}$ be conditionally convergent. Then

(a) $\mathbf{P}=\left(\Sigma a_{i}\right)^{\times} \circ\left(\Sigma a_{i}\right)^{\times}$,

(b) $\mathbf{P}_{0}=\left\{\pi \in \mathbf{P}:\left(\Sigma a_{i}\right)^{\times} \circ \pi \subseteq\left(\Sigma a_{i}\right)^{\times}\right\}$.

Proof. (a) Let $\pi \in \mathbf{P}$, and let $J$ be an infinite subset of $\mathbb{N}$ on which $\Sigma a_{i}$ converges absolutely. We show that $\pi=\rho_{1} \circ \rho_{2}$ where $\rho_{1}^{-1}$ and $\rho_{2}^{-1}$ are monotone on $\mathbb{N} \backslash J$. By Lemma $6, \rho_{1}$ and $\rho_{2}$ are in $\left(\Sigma a_{i}\right)^{\times}$.

Define $\rho_{1}^{-1}$ as follows: on $\mathbb{N} \backslash J, \rho_{1}^{-1}$ is the monotone bijection onto $J$. On $J, \rho_{1}^{-1}$ is the bijection onto $\mathbb{N} \backslash J$ such that, if $i, k \in J$, then $\rho_{1}^{-1}(i)<\rho_{1}^{-1}(k)$ if and only if $\pi^{-1}(i)<\pi^{-1}(k)$. Define $\rho_{2}^{-1}$ as follows: on $\mathbb{N} \backslash J, \rho_{2}^{-1}$ is the monotone bijection onto $\pi^{-1}(J)$, and on $J, \rho_{2}^{-1}$ is the bijection onto $\pi^{-1}(\mathbb{N} \backslash J)$ such that $\pi=\rho_{1} \circ \rho_{2}$.

(b) Notice that $\mathbf{P}_{0} \subseteq\left\{\pi \in \mathbf{P}:\left(\Sigma a_{i}\right)^{\times} \circ \pi \subseteq\left(\Sigma a_{i}\right)^{\times}\right\}$. To show equality let $\pi \in \mathbf{P} \backslash \mathbf{P}_{0}$. We shall construct $\rho$ in $\mathbf{P}_{0}$ such that $\rho \circ \pi \notin\left(\Sigma a_{i}\right)^{\times}$, so in fact we shall prove the stronger result that $\mathbf{P}_{0}=\left\{\pi \in \mathbf{P}: \mathbf{P}_{0} \circ \pi \subseteq\left(\Sigma a_{i}\right)^{\times}\right\}$. The construction of $\rho$ is such that there is an increasing function $U$ where $\rho([1, U(i)])=[1, U(i)]$ for all $i$, and for any $n, \rho([1, n])$ is a union of no more than two intervals of $\mathbb{N}$.

Set $U(1)=0$. In general, having defined $U(k)$ and $\rho(i)$ for $i \leqslant U(k)$, let $M(k+1)>U(k)$ be such that

$$
\sum_{i=U(k)+1}^{M(k+1)} \max \left(a_{i}, 0\right) \geqslant k+1
$$

let $n(k+1)$ be such that $\pi([1, n(k+1)])$ is a union of at least $M(k+1)+1-U(k)$ intervals of $\mathbb{N}$, the first of which contains $[1, U(k)]$; let $j_{U(k)+1}, \ldots, j_{M(k+1)}$ be the smallest elements of the second through $(M(k+1)+1-U(k))$ th intervals, in order; let

$$
N(k+1)=\max \{\pi(i): i \leqslant n(k+1)\} ;
$$

and let $U(k+1)$ and $L(k+1)$ be such that

$$
\begin{gathered}
L(k+1)>M(k+1), \\
U(k+1)=L(k+1)+N(k+1)-M(k+1)-1,
\end{gathered}
$$

and

$$
\sum_{i-L(k+1)}^{U(k+1)}\left|a_{i}\right|<1 /(k+1) \text {. }
$$

Define $\rho^{-1}(i)$ for $U(k)+1 \leqslant i \leqslant M(k+1)$ by

$$
\rho^{-1}(i)= \begin{cases}j_{i} & \text { if } a_{i}>0, \\ j_{i}-1 & \text { otherwise. }\end{cases}
$$

Define $\rho^{-1}(i)$ for $L(k+1) \leqslant i \leqslant U(k+1)$ so that it is monotone onto $[U(k)+1, N(k+1)] \backslash \rho^{-1}([U(k)+1, M(k+1)])$, and on $M(k+1)<i<L(k+1)$ define $\rho^{-1}(i)$ to be $N(k+1)+i-M(k+1)$. Now $\rho$ is defined on $[1, U(k+1)]$, $\rho([1, U(k+1)])=[1, U(k+1)]$, and for any $n \leqslant U(k+1), \rho([1, n])$ is a union of no more than two intervals of $\mathbb{N}$.

When the induction is complete, $\rho$ is defined. To show that $\rho \circ \pi \notin\left(\sum a_{i}\right)^{\times}$, consider

$$
\stackrel{n(k)}{\sum} a_{\rho \circ \pi(i)}
$$


for $k>1$. Now $\pi([1, n(k)])$ is composed of at least $M(k)+1-U(k-1)$ intervals. If $I(k)$ is the largest element of the first interval, then (*) contains $\Sigma^{I(k)} a_{\rho(i)}$. Since $\rho \in \mathbf{P}_{0}$, this sum converges as $k \rightarrow \infty$. The remaining terms in (*) can be grouped as

$$
\Sigma\left\{a_{i}: U(k-1)<i \leqslant M(k), a_{i}>0\right\}+\Sigma\left\{a_{i}: L(k) \leqslant i \leqslant U(k), \rho^{-1}(i) \in \pi([1, n(k)])\right\} .
$$

The second sum tends to 0 as $k \rightarrow \infty$, while the first sum tends to $\infty$. Hence (*) diverges.

We now answer Levi's question by showing that there are only two closed semigroups of permutations.

\section{Corollary 8. The only closed semigroups of permutations are $\mathbf{P}$ and $\mathbf{P}_{\mathbf{0}}$.}

Proof. Suppose that $P$ is closed and $\mathbf{P} \supset P \supset \mathbf{P}_{\mathbf{0}}$. Let $\pi \in P \backslash \mathbf{P}_{0}$. Since $P \neq \mathbf{P}$ and $P$ is closed, there is a conditionally convergent series $\Sigma a_{i}$ in $P^{+}$. As was mentioned at the start of the proof of Theorem 7(b), there is $\rho \in \mathbf{P}_{0} \subset P$ such that $\rho \circ \pi \notin\left(\Sigma a_{i}\right)^{\times} \supset P$, so $P$ is not a semigroup.

Despite the fact that the semigroup generated by $\left(\Sigma a_{i}\right)^{\times}$is equal to that generated by $\left(\Sigma b_{i}\right)^{\times}$whenever $\Sigma a_{i}$ and $\Sigma b_{i}$ belong to $\mathbf{C} \backslash \mathbf{A C}$, it is nevertheless true that $\left(\Sigma a_{i}\right)^{\times}$characterizes $\Sigma a_{i}$ quite closely. The following theorem is a slight restatement of results of Katznelson and McGehee [4].

THEOREM 9. Let $S$ be a finite subset of $\mathbf{C}$. Then $S^{\times+}=\operatorname{span}(S \cup \mathbf{A C})$.

Corollary 10. Let $\Sigma a_{i} \in \mathbf{C}$. Then

$$
\left\{\pi: \pi \circ\left(\Sigma a_{i}\right)^{\times} \subseteq\left(\Sigma a_{i}\right)^{\times}\right\}=\left\{\pi: \Sigma\left|a_{\pi(i)}-a_{i}\right|<\infty\right\} .
$$

Proof. Theorem 9 shows that if $\Sigma b_{i} \in\left(\Sigma a_{i}\right)^{\times+}$, then there is a real number $r$ such that $\Sigma\left|b_{i}-r a_{i}\right|<\infty$. Suppose that $\pi$ is such that $\pi \circ\left(\Sigma a_{i}\right)^{\times} \subseteq\left(\Sigma a_{i}\right)^{\times}$. Since the identity is in $\left(\Sigma a_{i}\right)^{\times}$, we have $\pi \in\left(\Sigma a_{i}\right)^{\times}$, and therefore $\Sigma a_{\pi(i)} \in \mathbf{C}$. If $\Sigma a_{\pi(i)} \notin\left(\Sigma a_{i}\right)^{\times+}$, then there is $\rho \in\left(\Sigma a_{i}\right)^{\times}$such that $\Sigma a_{\pi(\rho(i))} \neq \Sigma a_{\pi(i)}=\Sigma a_{i}$, and hence $\pi \circ \rho \notin\left(\Sigma a_{i}\right)^{\times}$. Therefore $\Sigma a_{\pi(i)} \in\left(\Sigma a_{i}\right)^{\times+}$, so there is $r$ such that $\Sigma\left|a_{\pi(i)}-r a_{i}\right|<\infty$. We shall show that $r=1$. If $\Sigma a_{i} \in \mathbf{A C}$ then $r$ can be any value, including 1. Otherwise, let $M=\Sigma\left|a_{\pi(i)}-r a_{i}\right|$ and let $I(\varepsilon)=\left\{i: a_{i} \geqslant \varepsilon\right\}$. Then

$$
M \geqslant \sum_{i \in I(\varepsilon)}\left|a_{\pi(i)}-r a_{i}\right| \geqslant-\sum_{i \in I(\varepsilon)} a_{\pi(i)}+r \sum_{i \in I(\varepsilon)} a_{i} .
$$

Since $\sum_{i \in I(\varepsilon)} a_{\pi(i)} \leqslant \sum_{i \in I(\varepsilon)} a_{i}$, it follows that

$$
M \geqslant(r-1) \sum_{i \in I(\varepsilon)} a_{i}
$$

If $r \neq 1$ then the right-hand side tends to infinity as $\varepsilon \rightarrow 0$, so we must have $r=1$.

Conversely, if $\Sigma\left|a_{\pi(i)}-a_{i}\right|<\infty$ then it is easy to see that $\Sigma a_{\pi(i)}=\Sigma a_{i}$ and $\Sigma a_{\pi(i)} \in\left(\Sigma a_{i}\right)^{\times+}$. Hence for any $\rho$ in $\left(\Sigma a_{i}\right)^{\times}, \Sigma a_{\pi(\rho(i))}=\Sigma a_{\pi(i)}=\Sigma a_{i}$, and so $\pi \circ \rho \in\left(\Sigma a_{i}\right)^{\times}$.

$$
\text { 4. } \pi^{+\times}
$$

In this section we consider the lattice structure of the small closed sets of permutations, a structure which is significantly more complicated than the small 
closed sets of series. The small closed sets of series are precisely characterized by Theorem 9, which converts lattice questions into linear algebra problems. For example, let $A$ be a closed subset of $\mathbf{C}$. Then any maximal descending chain of closed sets whose first element is $A$ and whose last element is $\mathbf{A C}$ has $\operatorname{dim}(A)+1$ members, where by $\operatorname{dim}(A)$ we mean the $\mathbb{N} \cup\{\infty\}$-valued linear space dimension of $A$ over AC. Also, given series $\Sigma a_{i}$ and $\Sigma b_{i}$ in $\mathbf{C}$ which are linearly independent over $\mathbf{A C}$, the closed sets strictly between $\left\{\Sigma a_{i}, \Sigma b_{i}\right\}^{\times+}$and $\mathbf{A C}$ are an antichain. Further, there is a bijection $f$ between $\mathbb{R} \cup\{\infty\}$ and these sets, where $f$ is given by

$$
f(r)= \begin{cases}\left\{\Sigma\left(a_{i}+r b_{i}\right)\right\}^{\times+}, & r \neq \infty, \\ \left(\Sigma b_{i}\right)^{\times+}, & r=\infty .\end{cases}
$$

Compared to such precise information, the results of this section are fairly crude. We shall show that if $\pi \in P \backslash \mathbf{P}_{0}$, then between $\pi^{+\times}$and $\mathbf{P}_{0}$ there are large chains and antichains. Thus to a certain degree the gross features of the lattice beneath $\pi^{+\times}$are independent of $\pi$. The following theorem starts this analysis. There are shorter proofs of the existence of $\rho_{2}$, but its method of construction is reused later.

TheOREM 11. Let $\pi$ be an element of $\mathbf{P} \backslash \mathbf{P}_{0}$. Then there are permutations $\rho_{1}$ and $\rho_{2}$ such that

$$
\mathbf{P} \supset \rho_{1}^{+x} \supset \pi^{+x} \supset \rho_{2}^{+x} \supset \mathbf{P}_{0} .
$$

Proof. We make extensive use of the fact that for any permutations $\rho$ in $\mathbf{P}$ and $\sigma$ in $\mathbf{P}_{0}, \rho \circ \sigma \in \rho^{+\times}$.

To construct $\rho_{1}$, let $j_{1}<j_{2}<\ldots$ be such that $\pi\left(j_{1}\right)<\pi\left(j_{2}\right)<\ldots$. Since $\mathbf{P}_{0}$ is not closed under inverses, there is a permutation $\sigma$ and series $\Sigma a_{i}$ in $\mathbf{C}$ such that $\sigma^{-1} \in \mathbf{P}_{\mathbf{0}}$ and $\sigma \notin\left(\Sigma a_{i}\right)^{\times}$. Define a new permutation $\tau$ by

$$
\tau(i)= \begin{cases}j_{\sigma(k)} & \text { if } i=j_{k}, \\ i & \text { otherwise. }\end{cases}
$$

Let $\rho_{1}=\pi \circ \tau$. Since $\pi=\rho_{1} \circ \tau^{-1}$ and $\tau^{-1} \in \mathbf{P}_{0}$, we have $\pi \in \rho_{1}^{+\times}$and $\pi^{+x} \subseteq \rho_{1}^{+x}$. To establish that $\pi^{+\times} \neq \rho_{1}^{+\times}$, we shall show that the series $\Sigma b_{i}$ defined below is in $\pi^{+} \backslash \rho_{1}^{+}$.

Define $\Sigma b_{i}$ by

$$
b_{i}= \begin{cases}a_{k} & \text { if } i=\pi\left(j_{k}\right), \\ 0 & \text { otherwise. }\end{cases}
$$

For any $n$ let $K(n)=\max \left\{k: \pi\left(j_{k}\right) \leqslant n\right\}$. Then

$$
\Sigma^{n} b_{i}=\Sigma^{K(n)} a_{i},
$$

so $\Sigma b_{i} \in \mathbf{C}$. Further, if $m(n)=\max \left\{k: j_{k} \leqslant n\right\}$, then

so $\Sigma b_{i} \in \pi^{+}$. However,

$$
\Sigma^{n} b_{\pi(i)}=\Sigma^{m(n)} a_{i},
$$

$$
\Sigma^{n} b_{\rho_{1}(i)}=\Sigma^{n} b_{\pi(\tau(i))}=\Sigma^{m(n)} a_{\sigma(i)} .
$$

Since $\sigma \notin\left(\Sigma a_{i}\right)^{\times}$and the range of $m(n)$ is $\mathbb{N}, \rho_{1} \notin\left(\Sigma b_{i}\right)^{+}$, as was to be shown.

To construct $\rho_{2}$ we shall find $\sigma$ in $\mathbf{P}_{0}$ such that $\pi \circ \sigma \notin \mathbf{P}_{0}$ and such that $(\pi \circ \sigma)^{+} \backslash \pi^{+}$is non-empty, for then $\rho_{2}=\pi \circ \sigma$ is the desired permutation. We define $\sigma$ by partitioning $\mathbb{N}$ into consecutive intervals $\left\{N_{n}\right\}_{n-1}^{\infty}$ such that $\sigma$ maps each $N_{n}$ onto 
itself. When $n$ is odd, $\sigma$ is the identity on $N_{n}$, while when it is even, then for any $i$ in $N_{n}, \sigma([1, i])$ is a union of not more than two intervals of $\mathbb{N}$. This ensures that $\sigma \in \mathbf{P}_{0}$. Let $m(n)=\max \left\{i: i \in N_{n}\right\}$ and $m^{\pi}(n)=\max \left\{\pi(i): i \in N_{n}\right\}$.

To define $\sigma$ and $\left\{N_{n}\right\}$, let $N_{1}=\{1\}$ and $\sigma(1)=1$. In general, having defined $N_{n-1}$, define $N_{n}$ as follows. Since $\pi \notin \mathbf{P}_{0}$, there is $K(n)>m(n-1)$ such that $\pi([1, K(n)]) \supset\left[1, m^{\pi}(n-1)\right]$ and $\pi([1, K(n)])$ consists of $n+1$ or more intervals. If $n$ is odd, let $N_{n}=[m(n-1)+1, K(n)]$ and define $\sigma$ to be the identity on $N_{n}$. If $n$ is even, let $j(n, k), 1 \leqslant k \leqslant n$, be such that

$$
m^{\pi}(n-1)<j(n, 1)<j(n, 2)<\ldots<j(n, n) \leqslant K(n)
$$

and $\pi(j(n, k))+1 \notin \pi([1, K(n)])$. (It is always possible to find such $j(n, k)$ since $\pi([1, K(n)])$ consists of $n+1$ or more intervals, the first of which includes $\left[1, m^{\pi}(n-1)\right]$.) Let $j^{\prime}(n, k), 1 \leqslant k \leqslant n$, be an increasing (in $k$ ) enumeration of $\left\{\pi^{-1}(\pi(j(n, 1))+1), \ldots, \pi^{-1}(\pi(j(n, n))+1)\right\}$. Notice that $j^{\prime}(n, 1)>K(n)$. Let

$$
N_{n}=\left[m(n-1)+1, j^{\prime}(n, n)\right] \text {. }
$$

Now $\sigma$ is defined on $N_{n}$ so that $\sigma(m(n-1)+1), \ldots, \sigma\left(j^{\prime}(n, n)\right)$ is

$$
\begin{aligned}
& m(n-1)+1, m(n-1)+2, \ldots, j(n, 1), j(n, n)+1, j(n, n)+2, \ldots, \\
& j^{\prime}(n, 1), j(n, 1)+1, j(n, 1)+2, \ldots, j(n, 2), j^{\prime}(n, 1)+1, j^{\prime}(n, 1)+2, \ldots, \\
& j^{\prime}(n, 2), j(n, 2)+1, \ldots, j(n, 3), j^{\prime}(n, 2)+1, \ldots, j^{\prime}(n, n) .
\end{aligned}
$$

The important feature is that, among $\left\{j(n, 1), \ldots, j(n, n), j^{\prime}(n, 1), \ldots, j^{\prime}(n, n)\right\}, \sigma$ lists them in the order $j(n, 1), j^{\prime}(n, 1), j(n, 2), j^{\prime}(n, 2), \ldots$.

As promised, $\sigma$ is in $\mathbf{P}_{0}$. To see that $\rho_{2} \notin \mathbf{P}_{0}$, notice that if $n$ is odd, then $\rho_{2}([1, m(n)])=\pi([1, m(n)])$, which consists of $n+1$ intervals. To see that $\rho_{2}^{+\times} \neq \pi^{+\times}$, define the series $\Sigma a_{i}$ by

$$
a_{i}= \begin{cases}1 / n & \text { if } i=\pi(j(n, k)) \text { for some } 1 \leqslant k \leqslant n, \\ -1 / n & \text { if } i=\pi\left(j^{\prime}(n, k)\right) \text { for some } 1 \leqslant k \leqslant n, \\ 0 & \text { otherwise. }\end{cases}
$$

By construction of $j$ and $j^{\prime}$, if one ignores the terms which are zero, then $\Sigma a_{i}$ is an alternating series, and hence converges, and its sum is 0 . To show that $\Sigma a_{i}$ is not in $\pi^{+}$, if $n$ is even then

$$
\stackrel{K(n)}{\Sigma} a_{\pi(i)}=\stackrel{m(n-1)}{\Sigma} a_{\pi(i)}+\sum_{i-m(n-1)+1}^{K(n)} a_{\pi(i)}=0+n \frac{1}{n}=1,
$$

so $\Sigma a_{\pi(i)}$ does not converge to $\Sigma a_{i}$. To see that $\Sigma a_{i} \in \rho_{2}^{+}$, let $l$ be the arbitrary and $n=\max \{j: m(j) \leqslant l\}$. Then

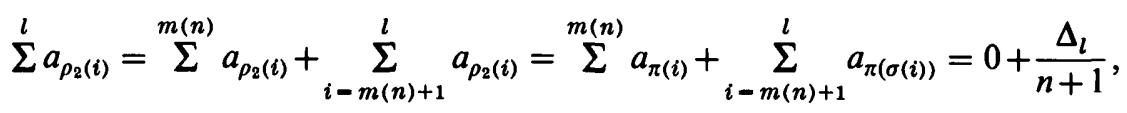

where $\Delta_{l}$ is either 0 or 1 , depending on whether

$$
\begin{aligned}
& \operatorname{card}(\{j(n+1,1), \ldots, j(n+1, n+1)\} \cap \sigma([m(n)+1, l])) \\
& \quad-\operatorname{card}\left(\left\{j^{\prime}(n+1,1), \ldots, j^{\prime}(n+1, n+1)\right\} \cap \sigma[(m(n)+1, l])\right)
\end{aligned}
$$

is equal to 0 or 1, respectively. Therefore $\Sigma a_{\rho_{2}(i)}=\Sigma a_{i}$, as was to be shown. 
By repeated application of Theorem 11 one obtains, for any $\pi \in P \backslash \mathbf{P}_{0}$, a countable decreasing chain $\pi^{+\times} \supset \pi_{1}^{+\times} \supset \pi_{2}^{+\times} \ldots$ of closed, singly generated sets of permutations. By manipulating the construction of $\rho_{2}$, longer chains can be produced. Recall that $\rho_{2}=\pi \circ \sigma$, where $\sigma \in \mathbf{P}_{0}$. The permutation $\sigma$ is constructed using $\left\{N_{n}\right\}$, where the behaviour of $\sigma$ on $\bigcup\left\{N_{n}: n\right.$ odd $\}$ guarantees that $\rho_{2} \notin \mathbf{P}_{0}$, while the behaviour of $\sigma$ on $\bigcup\left\{N_{n}: n\right.$ even $\}$ ensures that $\rho_{2}^{+\times} \neq \pi^{+\times}$. For a subset $S$ of the even integers, define the permutations $\sigma^{S}$ by

$$
\sigma^{S}(i)= \begin{cases}\sigma(i) & \text { if } i \in N_{n} \text { and } n \in S, \\ i & \text { otherwise. }\end{cases}
$$

It is easy to verify the following properties:

1. $\sigma=\sigma^{T}$ if $T=\{2,4,6, \ldots\}$;

2. $\sigma^{S} \in \mathbf{P}_{0}$ for all $S$;

3. $\pi \circ \sigma^{\phi}=\pi$;

4. $\left(\pi \circ \sigma^{S}\right)^{+\times} \supset\left(\pi \circ \sigma^{T}\right)^{+\times}$if $S \subset T$;

5. $\pi \circ \sigma^{S} \notin \mathbf{P}_{0}$ for all $S$;

6. $\left(\pi \circ \sigma^{S}\right)^{+\times}=\left(\pi \circ \sigma^{T}\right)^{+\times}$if and only if $(S \cup T) \backslash(S \cap T)$ is finite;

7. $\left(\pi \circ \sigma^{S}\right)^{+\times}$and $\left(\pi \circ \sigma^{T}\right)^{+\times}$are incomparable if $(S \backslash T)$ and $(T \backslash S)$ are infinite.

We can now describe some of the structure of the lattice between $\pi^{+\times}$and $\mathbf{P}_{\mathbf{0}}$.

Corollary 12. Let $\pi$ be an element of $\mathbf{P} \backslash \mathbf{P}_{0}$. Then the lattice of closed sets of permutations between $\pi^{+\times}$and $\mathbf{P}_{0}$ has large chains and antichains, in that

(a) there are permutations $\pi_{r}, 0 \leqslant r \leqslant 1$, such that $\pi_{0}=\pi$ and $\pi_{r}^{+\times} \supset \pi_{s}^{+\times}$ whenever $r<s$;

(b) there are permutations $\rho_{r}, 0 \leqslant r \leqslant 1$, such that $\pi^{+\times} \supset \rho_{r}^{+\times} \supset \mathbf{P}_{0}$ for all $r$ and $\rho_{r}^{+\times}$and $\rho_{s}^{+\times}$are incomparable whenever $r \neq s$.

Proof. Using the construction given above, we need only find appropriate subsets of the even integers. It is well known that there is a collection of sets $S_{r}$, $0 \leqslant r \leqslant 1$, such that each is a subset of the even integers, $S_{0}$ is all even integers, $S_{1}$ is infinite, and if $r<s$, then $S_{r} \supset S_{s}$ and $S_{r} \backslash S_{s}$ is infinite. Letting $\pi_{r}=\pi \circ \sigma^{S_{r}}$ gives the desired permutations. Similarly, it is well known that there are subsets $T_{r}$, $0 \leqslant r \leqslant 1$, of the even integers such that $T_{r} \backslash T_{s}$ and $T_{s} \backslash T_{r}$ are both infinite whenever $r \neq s$. Let $\rho_{r}=\pi \circ \sigma^{T}$.

Since there are only $c=\operatorname{card}(\mathbb{R})$ permutations, the chains and antichains of Corollary 12 are as large as possible if we consider only closed sets generated by a single permutation. It is possible that there are $2^{c}$ closed sets of permutations, in which case one can ask if a chain of that length is possible.

The meet of small closed sets behaves quite differently depending on whether the closed sets are sets of series or of permutations. On the one hand, Theorem 9 shows that for any series $\Sigma a_{i}$ and $\Sigma b_{i}$, either $\left(\Sigma a_{i}\right)^{x+}=\left(\Sigma b_{i}\right)^{x+}$ or else 
$\left(\Sigma a_{i}\right)^{x+} \wedge\left(\Sigma b_{i}\right)^{x+}=\mathbf{A C}$. On the other hand, the following theorem shows that almost anything is possible with permutations.

TheOREM 13. There are permutations $\pi_{1}, \pi_{2}, \pi_{3}, \pi_{4}, \rho_{1}, \rho_{2}, \rho_{3}, \rho_{4}$ in $\mathbf{P} \backslash \mathbf{P}_{0}$ such that

(a) $\pi_{1} \neq \rho_{1}$ but $\pi_{1}^{+\times}=\rho_{1}^{+\times} \supset \mathbf{P}_{0}$;

(b) $\pi_{2}^{+\times} \supset \pi_{2}^{+\times} \wedge \rho_{2}^{+\times}=\rho_{2}^{+\times} \supset \mathbf{P}_{0}$;

(c) $\pi_{3}^{+\times}$and $\rho_{3}^{+\times}$are incomparable (in which case $\pi_{3}^{+\times} \wedge \rho_{3}^{+\times}$is strictly smaller than either $\pi_{3}^{+\times}$or $\left.\pi_{3}^{+\times}\right)$and $\pi_{3}^{+\times} \wedge \rho_{3}^{+\times} \supset \mathbf{P}_{0}$;

(d) $\pi_{4}^{+\times}$and $\rho_{4}^{+\times}$are incomparable and $\pi_{4}^{+\times} \wedge \rho_{4}^{+\times}=\mathbf{P}_{0}$.

Proof. (a) Let $\pi_{1} \in \mathbf{P} \backslash \mathbf{P}_{0}$ be arbitrary, and let $\sigma \in \mathbf{P}_{0}$ be such that $\sigma$ is not the identity and $\sigma^{-1} \in \mathbf{P}_{0}$. Then $\rho_{1}=\pi_{1} \circ \sigma \in \pi_{1}^{+\times}$and $\pi_{1}=\rho_{1} \circ \sigma^{-1} \in \rho_{1}^{+x}$.

(b) This follows from Theorem 11 .

(c) Let $S$ and $T$ be infinite subsets of even numbers such that $S \backslash T$ and $T \backslash S$ are infinite. Using the notation preceeding Corollary 12, let $\pi_{3}=\pi \circ \sigma^{S}$ and $\rho_{3}=\pi \circ \sigma^{T}$. It is easy to see that $\pi_{3}^{+\times} \wedge \rho_{3}^{+\times}$is neither $\pi_{3}^{+\times}$nor $\rho_{3}^{+\times}$, and since it contains $\pi \circ \sigma^{S \cup T}$ it is not $\mathbf{P}_{\mathbf{0}}$.

(d) We define $\pi_{4}$ and $\rho_{4}$ on consecutive intervals of integers $B_{1}, B_{2}, \ldots$, where $B_{1}=[1,4], B_{2}=[5,10], B_{3}=[11,18]$, etc. The values of $\pi_{4}$ and $\rho_{4}$ are as indicated below.

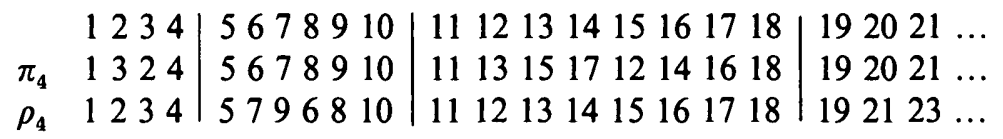

On even numbered blocks $\pi_{4}$ is the identity, while on odd numbered ones it lists first the odd elements and then the even ones; $\rho_{4}$ behaves similarly, exchanging the roles of odd and even blocks. Clearly $\pi_{4}, \rho_{4} \in \mathbf{P} \backslash \mathbf{P}_{0}$.

To show that $\pi_{4}^{+\times} \wedge \rho_{4}^{+\times}=\mathbf{P}_{0}$, notice that $\pi_{4}^{+\times} \wedge \rho_{4}^{+\times}=\left(\pi_{4}^{+} \cup \rho_{4}^{+}\right)^{\times}$, so it suffices to show that $\mathbf{C}=\operatorname{span}\left(\pi_{4}^{+} \cup \rho_{4}^{+}\right)$. Let $\Sigma a_{i} \in \mathbf{C}$ and let $b_{j}=\left(\Sigma_{i \in B_{j}} a_{i}\right) / \operatorname{card}\left(B_{j}\right)$. Define series $\Sigma c_{i}, \Sigma d_{i}$ by

$$
c_{i}=\left\{\begin{array}{ll}
2 a_{i}-b_{j} & \text { if } i \in B_{j} \text { and } j \text { even, } \\
b_{j} & \text { if } i \in B_{j} \text { and } j \text { odd, }
\end{array} \quad d_{i}= \begin{cases}2 a_{i}-b_{j} & \text { if } i \in B_{j} \text { and } j \text { odd, } \\
b_{j} & \text { if } i \in B_{j} \text { and } j \text { even. }\end{cases}\right.
$$

Then $2 a_{i}=c_{i}+d_{i}$ for all $i$, so $\Sigma a_{i}$ is in the span of $\Sigma c_{i}$ and $\Sigma d_{i}$. To show that $\Sigma c_{i}, \Sigma d_{i} \in \mathbf{C}$, notice that for any block $B_{j}$,

$$
\sum_{i \in B_{j}} c_{i}=\sum_{i \in B_{j}} d_{i}=\sum_{i \in B_{j}} a_{i}
$$

Pick any $n$, let $j$ be the number of the block containing $n$, and let $m(n)=\max B_{j-1}$. Then

$$
\stackrel{n}{\Sigma}^{n} c_{i}=\sum^{m(n)} c_{i}+\sum_{m(n)+1}^{n} c_{i}=\sum^{m(n)} a_{i}+\sum_{m(n)+1}^{n} c_{i} .
$$

To estimate $\left|\Sigma_{m(n)+1}^{n} c_{i}\right|$, notice that if $j$ is odd then this is equal to $\left|(n-m(n)) b_{j}\right|$; this is less than or equal to $\left|\Sigma_{i \in B_{j}} a_{i}\right|$, which tends to 0 as $n$ tends to infinity. If $j$ is even then

$$
\left|\sum_{m(n)+1}^{n} c_{i}\right|=\left|\sum_{i-m(n)+1}^{n} 2 a_{i}-b_{j}\right| \leqslant 2\left|\sum_{i-m(n)+1}^{n} a_{i}\right|+(n-m(n))\left|b_{j}\right|,
$$


which also tends to 0 as $n$ tends to infinity. Therefore $\Sigma c_{i}$ converges to the sum $\Sigma a_{i}$. Since $c_{i}=c_{\pi_{4}(i)}$ for all $i$, we also have $\Sigma c_{i} \in \pi_{4}^{+}$. A similar proof shows that $\Sigma d_{i} \in \rho_{4}^{+}$.

For obvious reasons we have been calling closed sets of the form $\left(\sum a_{i}\right)^{x+}$ and $\pi^{\times+}$ small, and the following simple proposition gives another measure of their smallness.

Proposition 14. (a) Let $\left\{\pi_{n}: n \in \mathbb{N}\right\}$ be a countable collection of permutations. Then $\bigvee_{n} \pi_{n}^{+\times} \subset \mathbf{P}$.

(b) Let $\left\{\Sigma a_{i}^{n}: n \in \mathbb{N}\right\}$ be a countable collection of convergent series. Then $\bigvee_{n}\left(\Sigma a_{i}^{n}\right)^{\times+} \subset \mathbf{C}$.

Proof. (a) We define a function $j(n, i), i, n \in \mathbb{N}$, such that $j(n, i)$ is strictly increasing in $i$ for each fixed $n$. First, let $j(1, i)$ be an increasing sequence such that $\pi_{1}^{-1}(j(1,1))<\pi_{1}^{-1}(j(1,2))<\ldots$. Having defined $j(n-1, i)$ for some $n$, let $j(n, i)$ be an increasing (in $i$ ) subsequence of $j(n-1, i)$ such that $\pi_{n}^{-1}(j(n, 1))<\pi_{n}^{-1}(j(n, 2))<\ldots$. Finally, define the function $k(i)$ by $k(i)=j(i, i)$. By construction, $k$ is increasing, and for any $n, \pi_{n}^{-1}(k(i))$ is increasing when $i \geqslant n$.

To show that $\bigvee \pi_{n}^{+\times} \neq \mathbf{P}$, it suffices to find a conditionally convergent series $\Sigma b_{n}$ in $\bigcap \pi_{n}^{+}$. The series

$$
b_{i}= \begin{cases}(-1)^{j} / j & \text { if } i=k(j), \\ 0 & \text { otherwise }\end{cases}
$$

will do.

(b) By a diagonalization process similar to that used above, one can find an infinite subset $S \subset \mathbb{N}$ such that $\Sigma a_{i}^{n}$ converges absolutely on $S$ for all $n$. Therefore any permutation $\pi$ which is the identity on $\mathbb{N} \backslash S$ is in $\bigcap\left(\Sigma a_{i}^{n}\right)^{\times}$, and thus $\bigvee\left(\Sigma a_{i}^{n}\right)^{\times+}$omits any series $\Sigma b_{i}$ in $\mathbf{C} \backslash \mathbf{A C}$ where $b_{i}=0$ for $i \in \mathbb{N} \backslash S$.

\section{Alternating series}

Perhaps the most important class of conditionally convergent series is the set of alternating series, where a series $\sum a_{i}$ is alternating if $a_{i} a_{i+1} \leqslant 0$ for all $i$ and $\left|a_{i}\right|$ converges monotonically to 0 . We use Alt to denote the set of alternating series. Now Alt is a well-known class of series with several nice properties, making it natural to consider Alt ${ }^{x+}$. To do this we shall first characterize Alt ${ }^{\times}$.

Let $S$ be a subset of $\mathbb{N}$ and let $\rho$ be the increasing enumeration of $S$, that is, $\rho(1)$ is the smallest element of $S, \rho(2)$ is the second smallest, etc. (If $S$ is a finite set then $\rho$ has domain $[1, \operatorname{card}(S)]$.) We say that $S$ is alternating if $\rho$ alternates between even and odd numbers. The imbalance of $S$ is defined to be

$$
\max \left\{\left|\sum_{l=1}^{i}(-1)^{\rho(l)}\right|: i \leqslant \operatorname{card}(S)\right\} .
$$

Notice that every non-empty set has a non-zero imbalance, and an alternating set has an imbalance of 1 . The converse is not true, as the set $\{1,2,4,5\}$ has imbalance 1 but does not alternate. The following facts concerning imbalance are straightforward and their proofs are left to the reader.

Proposition 15. Let $S$ be a subset of $\mathbb{N}$.

(a) If $S$ has an imbalance of 1 , then $S$ can be partitioned into two alternating sets, one of which may be empty. 
(b) If $S$ has an imbalance of $k$, then $S$ can be partitioned into $k$ sets each having an imbalance of 1 .

(c) If $S$ has an imbalance of $k$ and $S^{\prime} \subset S$ has an imbalance of 1 , then the imbalance of $S \backslash S^{\prime}$ is $k-1, k$, or $k+1$.

Combining parts (a) and (b) we see that a set of imbalance $k$ can be partitioned into $2 k$ alternating sets, some of which may be empty.

We extend the notion if imbalance to permutations by saying that the imbalance of a permutation $\pi$ is $\max _{n}$ \{imbalance of $\left.\pi([1, n])\right\}$. It is possible for a permutation $\pi$ to alternate in the sense that $\pi(1)$ is odd, $\pi(2)$ is even, $\pi(3)$ is odd, etc., and yet still have infinite imbalance. For example, the permutation with values $1,4,3,2,5,10$, $7,8,9,6,11,18,13,16, \ldots$ has these properties.

Proposition 16. Alt ${ }^{\times}=\{\pi: \pi$ has finite imbalance $\}$.

Proof. Let $\pi$ have a finite imbalance $I$ and let $\Sigma a_{i}$ be in Alt. Define the function $\pi^{-}$from $\mathbb{N}$ to $\mathbb{N} \cup\{0\}$ by

$$
\pi^{-}(n)=\min \{\mathbb{N} \backslash \pi([1, n])\}-1,
$$

that is, $\pi^{-}(n)$ is the largest $i$ such that $\pi([1, n])$ contains $[1, i]$. Then

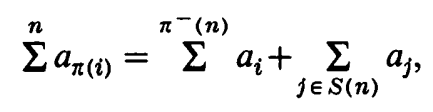

where $S(n)=\pi([1, n]) \backslash\left[1, \pi^{-}(n)\right]$. Since $\pi$ has an imbalance of $I . S(n)$ has an imbalance of at most $I+1$. Partition $S(n)$ into $2 I+2$ alternating sets $S_{1}, \ldots, S_{2 I+2}$.

For each non-empty alternating set $S_{j}$,

$$
\left|\sum_{i \in S_{j}} a_{i}\right| \leqslant\left|a_{k(j)}\right|
$$

where $k(j)=\min S_{j}$. Since $k(j)>\pi^{-}(n)$, we have $\left|a_{k(j)}\right| \leqslant\left|a_{\pi^{-}(n)}\right|$, so

$$
\left|\sum_{j \in S(n)} a_{i}\right| \leqslant \sum_{j=1}^{2 I+2}\left|\sum_{i \in S_{j}} a_{i}\right| \leqslant(2 I+2) \cdot\left|a_{\pi}{ }^{-}(n)\right| .
$$

This tends to 0 as $n$ tends to infinity, so upon substituting this into (**) we see that $\Sigma a_{\pi(i)}=\Sigma a_{i}$. Therefore $\pi \in \mathbf{A l t}^{\times}$.

To prove the converse, suppose that $\pi$ has infinite imbalance. Inductively define two functions $n$ and $m$ by setting $n(1)=0$ and $m(1)=2$, and having defined $n(k)$ and $m(k)$, pick $n(k+1)$ and $m(k+1)$ such that

(i) $n(i+1)$ and $m(k+1)$ are even,

(ii) $n(k+1)>n(k)$ and $m(k+1)>m(k)$,

(iii) $\pi([1, m(k+1)]) \supset[1, n(k)]$,

(iv) $\left|\Sigma\left\{(-1)^{i}: i \in \pi([1, m(k+1)]), n(k)<i \leqslant n(k+1)\right\}\right| \geqslant m(k)^{2}$.

(One can always find $n(k+1)$ and $m(k+1)$ satisfying (i) through (iii), and (iv) can also be satisfied since $\pi$ has infinite imbalance.) 
Define a series $\Sigma a_{i}$ in Alt by $a_{i}=(-1)^{i} / m(k-1)$, where $k=\min \{j: i \leqslant n(j)\}$. Since $n(k)$ is always even, $a_{i}=-a_{i+1}$ when $i$ is odd, and $\Sigma^{n(k)} a_{i}=0$. Let $k>1$. Then

$$
\begin{aligned}
\stackrel{m(k)}{\sum} a_{\pi(i)} & =\sum^{n(k-1)} a_{i}+\Sigma\left\{a_{\pi(i)}: i \leqslant m(k), \pi(i)>n(k-1)\right\} \\
& =0+\sum_{i \in S}(-1)^{i} / m(k-1)+\sum_{i \in T}(-1)^{i} / m(k),
\end{aligned}
$$

where $S=\{i: i \in \pi([1, m(k)]), n(k-1)<i \leqslant n(k)\}$ and $T=\{i: i \in \pi([1, m(k)]), i>n(k)\}$. By property (iv), the summation over $S$ has absolute value at least $m(k-1) ; T$ has no more than $m(k)$ elements, so the sum over $T$ has absolute value no greater than 1 . Therefore ( $\$)$ tends to infinity as $k$ tends to infinity, so $\pi \notin$ Alt $^{\times}$, as was to be shown.

Before characterizing Alt ${ }^{x+}$ we first need a small technical fact.

Lemma 17. Suppose that $\Sigma a_{i}$ is such that $\lim a_{i}=0$ and $\sum\left|a_{i}+a_{i+1}\right|<\infty$. Then there are series $\Sigma b_{i}$ and $\Sigma c_{i}$ in Alt such that $a_{i}=b_{i}+c_{i}$ for alt $i$.

Proof. Define $\Delta_{i}$ by $\Delta_{i}=1$ if $i$ is odd and $a_{i} \geqslant 0$ or $i$ is even and $a_{i}<0$, and $\Delta_{i}=0$ otherwise. Define $\Sigma b_{i}$ and $\Sigma c_{i}$ by

$$
\begin{gathered}
b_{i}=(-1)^{i+1}\left[\left|a_{i}\right| \Delta_{i}+\sum_{j=i}^{\infty}\left|a_{j}+a_{j+1}\right|\right], \\
c_{i}=(-1)^{i}\left[\left|a_{i}\right|\left(1-\Delta_{i}\right)+\sum_{j=i}^{\infty}\left|a_{j}+a_{j+1}\right|\right] .
\end{gathered}
$$

Clearly $b_{i}+c_{i}=a_{i}$, both $\Sigma b_{i}$ and $\Sigma c_{i}$ have alternating signs, and $\lim _{i} b_{i}=\lim _{i} c_{i}=0$. To finish we need only show that $\left|b_{i}\right|$ and $\left|c_{i}\right|$ converge monotonically. We do this for $\left|b_{i}\right|$ only, the $\left|c_{i}\right|$ case being identical. We have

$$
\left|b_{i}\right|-\left|b_{i+1}\right|=\left|a_{i}+a_{i+1}\right|+\Delta_{i}\left|a_{i}\right|-\Delta_{i+1}\left|a_{i+1}\right| .
$$

If $a_{i} a_{i+1} \leqslant 0$, then $\Delta_{i}=\Delta_{i+1}$ and $(\xi)$ is equal to 0 or $\left|a_{i}+a_{i+1}\right|$. Otherwise, $\left|a_{i}+a_{i+1}\right|=\left|a_{i}\right|+\left|a_{i+1}\right|$, in which case (घ) gives

$$
\left|b_{i}\right|-\left|b_{i+1}\right|=\left|a_{i}\right|\left(1+\Delta_{i}\right)+\left|a_{i+1}\right| \cdot\left(1-\Delta_{i+1}\right) \geqslant 0 .
$$

Therefore $\left|b_{i}\right|$ is monotonic in $i$, and $\Sigma b_{i} \in$ Alt.

TheOrem 18. $\Sigma a_{i}$ is in $\mathbf{A l t} \times+$ if and only if $\lim a_{i}=0$ and $\Sigma\left|a_{i}+a_{i+1}\right|<\infty$.

Proof. Suppose that $\lim a_{i}=0$ and $\Sigma\left|a_{i}+a_{i+1}\right|<\infty$. Then, by Lemma 17, $\Sigma a_{i}$ is in the linear span of Alt, and hence is in Alt ${ }^{\times+}$.

Conversely, suppose that $\Sigma a_{i} \in \mathbf{C}$ and $\Sigma\left|a_{i}+a_{i+1}\right|=\infty$; we shall show that $\Sigma a_{i} \notin \mathbf{A l t}{ }^{\times+}$. Since $\Sigma\left|a_{i}+a_{i+1}\right|=\infty$, either $\Sigma\left|a_{2 i-1}+a_{2 i}\right|=\infty, \Sigma\left|a_{2 i}+a_{2 i+1}\right|=\infty$, or both. We assume that the first holds, the second case being similar. If $b_{i}=a_{2 i-1}+a_{2 i}$ then $\Sigma b_{i}$ is conditionally convergent, so there is a permutation $\rho$ such that $\Sigma b_{\rho(i)}$ diverges. Define a permutation $\pi$ by

$$
\pi(2 i-1)=2 \rho(i)-1 \quad \text { and } \quad \pi(2 i)=2 \rho(i) .
$$


Now $\pi$ has imbalance 1 , so it is in Alt ${ }^{\times}$. However,

$$
\sum_{i=1}^{2 n} a_{\pi(i)}=\sum_{i=1}^{n} b_{\rho(i)}
$$

so $\sum a_{\pi(i)}$ diverges. Therefore $\left(a_{i}\right) \notin \mathbf{A l t}{ }^{x+}$.

COROLlaRY 19. $\Sigma a_{i} \in \mathbf{A l t} \times+$ if and only if there are series $\Sigma b_{i}, \Sigma c_{i}$ in Alt such that $a_{i}=b_{i}+c_{i}$ for all $i$.

There is another set of series which initially looks like a promising extension of Alt. Let $\mathbf{A}$ denote all series $\Sigma a_{i}$ such that when the zero terms are deleted, the resulting series is in Alt or has only finitely many terms. For example, $\left\{1,0,-\frac{1}{2}, 0, \frac{1}{3}, 0, \ldots\right\}$ is in A. Clearly, if $\Sigma a_{i}$ is in $\mathbf{A}$, then $\Sigma a_{i}$ converges, so one might hope that $\mathbf{A}^{\times+}$is a useful, non-trivial class of convergent series. Unfortunately, an examination of Levi's proof of Theorem 1 shows that $\mathbf{A}^{x+}=\mathbf{C}$, since he shows that for any $\pi$ in $\mathbf{P} \backslash \mathbf{P}_{\mathbf{0}}$ there is $\Sigma a_{i}$ in $\mathbf{A}$ such that $\pi \notin\left(\Sigma a_{i}\right)^{\times}$.

\section{Final remarks}

The mixture of duality, linearity of $\mathbf{C}$, and multiplication in $\mathbf{P}$ gives a very rich structure, only a small portion of which has been considered here. Many questions concerning the unstudied portions immediately suggest themselves, and we mention only a few. For example, Pleasants proved that the group generated by $\mathbf{P}_{\mathbf{0}}$ is not all of $\mathbf{P}$, while we have shown that the semigroup generated by $\left(\Sigma a_{i}\right)^{\times}$is always $\mathbf{P}$. What about closed sets between $\mathbf{P}_{0}$ and $\left(\Sigma a_{i}\right)^{\times}$? In particular, if $\pi \in \mathbf{P} \backslash \mathbf{P}_{0}$, what can one say about the group and semigroup generated by $\pi^{+\times}$?

Our most glaring omission is the lack of a characterization of $\pi^{+x}$. A sufficient condition for $\rho$ to be in $\pi^{+\times}$is that there is $N$ such that, for all $n$,

$$
\rho([1, n])=\bigcup_{k=1}^{N^{\prime}} I_{k} \backslash \bigcup_{k=1}^{N^{\prime \prime}} J_{k}
$$

where $N^{\prime}+N^{\prime \prime} \leqslant N$ and each $I_{k}$ and $J_{k}$ is either an interval of $\mathbb{N}$ or a ' $\pi$-interval' of the form $\pi([l, m])$ for some $l \leqslant m$. The presence of the $J$ terms may seem strange, but the following example shows why they are useful. Consider the permutations $\pi$ and $\rho$ given by:

$$
\begin{array}{ll|l|l|l} 
& 1,2,3,4,5,6,7,8 & 9,10,11,12,13,14,15,16,17,18,19 & 20,21,22,23 \ldots \\
\pi & 4,1,6,2,8,3,5,7 & 13,9,15,10,17,11,19,12,14,16,18 & 25,20,27,21 \ldots \\
\rho & 4,6,8,5,7,1,2,3 & 13,15,17,19,14,16,18,9,10,11,12 & 25,27,29,31 \ldots
\end{array}
$$

The vertical lines break $\mathbb{N}$ into blocks, where both $\pi$ and $\rho$ map each block onto itself. In the first block, $\rho$ and $\pi$ have the same order except $\rho$ saves $1 \ldots 3$ for last. In the second block $\rho$ saves $9 \ldots 12$, etc. Now $\rho \in \pi^{+\times}$and $\pi \in \rho^{+\times}$, and for any $n$, $\rho([1, n])=\pi([1, n+k]) \backslash[l, m]$ for some $k, l, m$ (if $k=0$ then the subtracted set is empty). To express $\rho([1,3])$ solely as a union takes at least 3 intervals and $\pi$-intervals; to express $\rho([1,12])$ takes at least 4 , etc. Incidentally, $\rho \in \pi^{+\times}$and $\pi \in \rho^{+\times}$and yet $\rho \neq \pi \circ \sigma$ and $\pi \neq \rho \circ \sigma$ for any $\sigma$ in $\mathbf{P}_{\mathbf{0}}$.

There is also the more general problem of characterizing $A^{\times+}$and $P^{+\times}$for sets $A \subset \mathbf{C}$ and $P \subset \mathbf{P}$. This may well be too difficult, in which case perhaps a more accessible problem is to characterize $A \vee B$ and $P \vee Q$ for closed sets $A, B \subset \mathrm{C}$ and $P, Q \subset \mathbf{P}$. 
We have shown that, if $\Sigma a_{i}$ and $\Sigma b_{i}$ are conditionally convergent, then $\left(\Sigma a_{i}\right)^{\times}$ and $\left(\Sigma b_{i}\right)^{\times}$have several semigroup properties in common, and that the lattices beneath $\left(\Sigma a_{i}\right)^{x+}$ and $\left(\Sigma b_{i}\right)^{x+}$ are (trivially) isomorphic. Is the lattice of closed sets larger than $\left(\Sigma a_{i}\right)^{x+}$ isomorphic to the closed sets larger than $\left(\Sigma b_{i}\right)^{x+}$ ? Similarly, is the lattice of closed sets smaller than $\pi^{+\times}$isomorphic to those smaller than $\rho^{+\times}$for all $\rho$ and $\pi$ in $\mathbf{P} \backslash \mathbf{P}_{0}$ ?

\section{References}

1. R. P. Agnew, 'Permutations preserving convergence of series', Proc. Amer. Math. Soc. 6 (1955) $563-564$.

2. A. Dvoretzky and C. A. Rogers, 'Absolute and unconditional convergence in normed linear spaces', Proc. Nat. Acad. Sci. U.S.A. 36 (1950) 192-197.

3. U. C. Guнa, 'On Levi's theorem on rearrangement of convergent series', Indian J. Math. 9 (1967) 91-93.

4. Y. KATZNELSON and O. C. MCGeHEE, 'Conditionally convergent series in $R^{\infty}$ ', Michigan Math. J. 21 (1974) 97-106.

5. F. W. LEVI, 'Rearrangement of convergent series', Duke Math. J. 13 (1946) 579-585.

6. P. A. B. Pleasants, 'Rearrangements that preserve convergence', J. London Math. Soc. (2) 15 (1977) $134-142$.

7. P. SCHAEFER, 'Sum-preserving rearrangements of infinite series', Amer. Math. Monthly 88 (1981) $33-40$.

8. J. H. SMITH, 'Rearrangements of conditionally convergent real series with preassigned cycle type', Proc. Amer. Math. Soc. 47 (1975) 167-170.

9. E. Steinitz, 'Bedingt konvergente Reihen and konvexe systeme', J. Reine Angew. Math. 143 (1913) 128-175; ibid. 144 (1914) 1-40.

10. W. Threlfall, 'Bedingt konvergente Reihen', Math. Z. 24 (1925) 212-214.

11. A. WALD, 'Vereinfachter Beweis des Steinitzschen Satzes über Vektorreihen im $R_{n}$ ', Ergebnisse Math. Kolloqu. 5 (1933) 10-13.

12. A. WALD, 'Bedingt konvergente Reihen von Vektoren im $R_{\omega}$ ', Ergebnisse Math. Kolloqu. 5 (1933) 13-14.

Department of Electrical Engineering and Computer Science The University of Michigan

Ann Arbor

Michigan 48109

USA 